\title{
Facile Formation of Anatase Nanoparticles on H-Titanate Nanotubes at Low Temperature for Efficient Visible Light-Driven Degradation of Organic Pollutants
}

\author{
Weiwei Fu ${ }^{1, *}$, Zhiqiang Shi ${ }^{2}$, Helong Bai ${ }^{1}$, Jinyu Dai ${ }^{2}$, Zhiming Lu ${ }^{2}$, Feifei Lei ${ }^{2}$, \\ Deguang Zhang ${ }^{2}$, Lun Zhao ${ }^{1, *}$ and Zongtao Zhang ${ }^{2, *}$ \\ 1 College of Chemistry, Changchun Normal University, Changchun 130032, China; baihelong@ccsfu.edu.cn \\ 2 State Key Laboratory of Inorganic Synthesis \& Preparative Chemistry, Jilin University, Changchun 130012, \\ China; shizq17@jlu.edu.cn (Z.S.); daijy16@mails.jlu.edu.cn (J.D.); zmlu18@mails.jlu.edu.cn (Z.L.); \\ leiff18@mails.jlu.edu.cn (F.L.); zhangdg17@mails.jlu.edu.cn (D.Z.) \\ * Correspondence: fuweiwei@ccsfu.edu.cn (W.F.); zhaolun@mail.cncnc.edu.cn (L.Z.); \\ zzhang@jlu.edu.cn (Z.Z.); Tel.: +86-431-8616-8099 (W.F.)
}

Received: 13 May 2020; Accepted: 17 June 2020; Published: 19 June 2020

\begin{abstract}
Anatase nanoparticles (5-10 nm) generated on H-titanate nanotube surface (H-titanate/anatase) were prepared by an ingenious and simple method. H-titanate tubes were prepared by a hydrothermal reaction of $\mathrm{Ti}$ powder in concentrated $\mathrm{NaOH}$ solution and an ion exchange process with $\mathrm{HNO}_{3}$ solution. After that, at a relatively low drying temperature $\left(100{ }^{\circ} \mathrm{C}\right)$, a small quantity of anatase nanoparticles were in-situ formed on the H-titanate tubes surface by a surface dehydration reaction. In-situ transformation can form a strong interface coupling between $\mathrm{H}$-titanate and anatase, which is conducive to accelerating charge transfer and improving its photocatalytic activity. In addition, the smaller average crystal size, the large specific surface areas (BET), the nanotubed and layered structure and the synergistic effect of dual phases would be beneficial to improving the photocatalytic efficiency.
\end{abstract}

Keywords: in-situ formation; anatase nanoparticles; H-titanate nanotubes; dual-phase; low temperature

\section{Introduction}

Water contamination resulting from the rapid development of industrialization has attracted worldwide attention. Photocatalytic degradation is the most promising strategy to completely solve the organic pollutants problem [1]. Among all kinds of conversion systems, $\mathrm{TiO}_{2}$-based materials for water pollution are considered to be an environmentally friendly and promising way to efficiently utilize solar energy [2,3]. Under UV irradiation, anatase can degrade a broad range of tenacious and toxic organic contaminants in water, and it is nontoxic, relatively cheap and chemically stable. However, its photocatalytic application is limited owing to the rapid recombination of the excited electron-hole pairs, the low visible light activity and low surface area [4,5]. Coupling anatase with another semiconductor favors a narrow band gap and the electron-hole separation [6-9], so as to improve the quantum efficiency. Moreover, the synergistic effect between two different phases can also enhance the photocatalytic activity $[6,10]$.

It was reported that $\mathrm{H}$-titanate was formed from $\mathrm{TiO}_{2}$ reacting with a concentrated $\mathrm{NaOH}$ solution to form titanate and then the ion exchange reacted with a dilute acid solution [11-13]. After calcination at a high temperature, $\mathrm{H}$-titanate can transform into anatase $\mathrm{TiO}_{2}$ [13]. Based on the above formation 
mechanism of $\mathrm{TiO}_{2}-\mathrm{H}$-titanate- $\mathrm{TiO}_{2}$, it is estimated that, at low calcination temperature, a small amount of anatase $\mathrm{TiO}_{2}$ will be formed in situ on the $\mathrm{H}$-titanate nanotube surface. Several papers have reported the synthesis of titanate/anatase composites; however, most preparation processes were carried out under high pressure and high temperature conditions, which consumed more energy [14-16]. For example, Xiong et al. reported a nitrogen-doped titanate-anatase core-shell nanobelts. In this paper, the titanate-anatase was obtained by calcination at $500{ }^{\circ} \mathrm{C}$ [14]. Yan et al. demonstrated that the titanate nanotube/anatase nanoparticle composites could be prepared by the hydrothermal method using as-obtained titanate tube dispersed into a $\mathrm{HNO}_{3}$ solution [15]. Herein, we report that a dual-phase photocatalyst (anatase nanoparticles $(5-10 \mathrm{~nm}$ ) was generated in situ on a H-titanate nanotube surface) was obtained via a controllable surface dehydration reaction at low temperature $\left(100{ }^{\circ} \mathrm{C}\right)$ and atmospheric pressure, which exhibited a higher visible light photocatalytic activity than P25; pure H-titanate nanotubes and pure anatase. The efficient visible light photocatalytic activity can be attributed to: (1) In situ transformation can form strong interfacial coupling between $\mathrm{H}$-titanate and anatase, which is favorable to accelerating charge transfer [17]; (2) H-titanate has a layered structure, which is composed of $\mathrm{TiO}_{6}$ octahedra sheets sharing four edges, similar to that of anatase crystals, which is easy to form a heterostructure between anatase and H-titanate [14,18,19]. In the meantime, at a low drying temperature, dual-phase catalyst retained the nanotubed and layered structures, which was beneficial for the high BET surfaces to adsorb organic pollutants and promote the diffusion of organic molecules inside the pores. The smaller average crystal size of anatase nanoparticles means a stronger redox ability in the photocatalytic process. Therefore, in the presence of the dual-phase catalyst, rhodamine $\mathrm{B}(\mathrm{RhB})$ and methylene blue $(\mathrm{MB})$ can be completely decomposed in a very short time under visible light irradiation. In addition, the synergistic effect would be beneficial to improve the photocatalytic efficiency.

\section{Results and Discussion}

Figure 1 shows the X-ray diffraction (XRD) patterns of the as-synthesized material. As shown in Figure $1 \mathrm{a}$, there are several diffraction peaks located at $2 \theta=9.8^{\circ}, 24.4^{\circ}, 28.4^{\circ}, 48.4^{\circ}$ suggesting that the as-prepared sample is layered titanate with a component of $\mathrm{H}_{2} \mathrm{Ti}_{2} \mathrm{O}_{4}(\mathrm{OH})_{2}$ [20] and a 0.9 nm interlayer distance, which is further confirmed by TEM observation. After drying at $100{ }^{\circ} \mathrm{C}$, the crystal structure of H-titanate was well maintained; the diffraction peaks of anatase-type $\mathrm{TiO}_{2}$ (JCPDS no. 21-1272) [21] are clearly observed (Figure 1b), showing that dual-phase H-titanate/anatase was obtained at a low temperature, which may be due to the dehydration of the H-titanate. The broad peaks indicate its low crystallinity and nanosized crystallites. With increasing calcination temperature, the peaks of anatase become narrower and sharper (Figure 1c,d). After calcination at $500{ }^{\circ} \mathrm{C}$ for $5 \mathrm{~h}$, all diffraction peaks of H-titanate disappeared (Figure 1e), which suggested that the layered H-titanate was completely transformed into anatase. The apparent sharpening of peaks suggests its high crystallinity. To further confirm the coexistence of these two $\mathrm{TiO}_{2}$ phases in the as-synthesized products, Raman spectroscopy was tested for the H-titanate and dual-phase catalysts (Figure 2). In Figure 2b, the peaks that center at $143\left(\mathrm{E}_{\mathrm{g}}\right), 514\left(\mathrm{~A}_{1 \mathrm{~g}}\right)$ and $636 \mathrm{~cm}^{-1}\left(\mathrm{E}_{\mathrm{g}}\right)$ belong to the anatase phase [22], while others match well with the hydrogen titanate phase (shown in Figure $2 a$ ). The Raman spectrum in Figure $2 b$ is composed of the characteristic peaks of the H-titanate and anatase phase, which are in good agreement with the XRD analysis. 

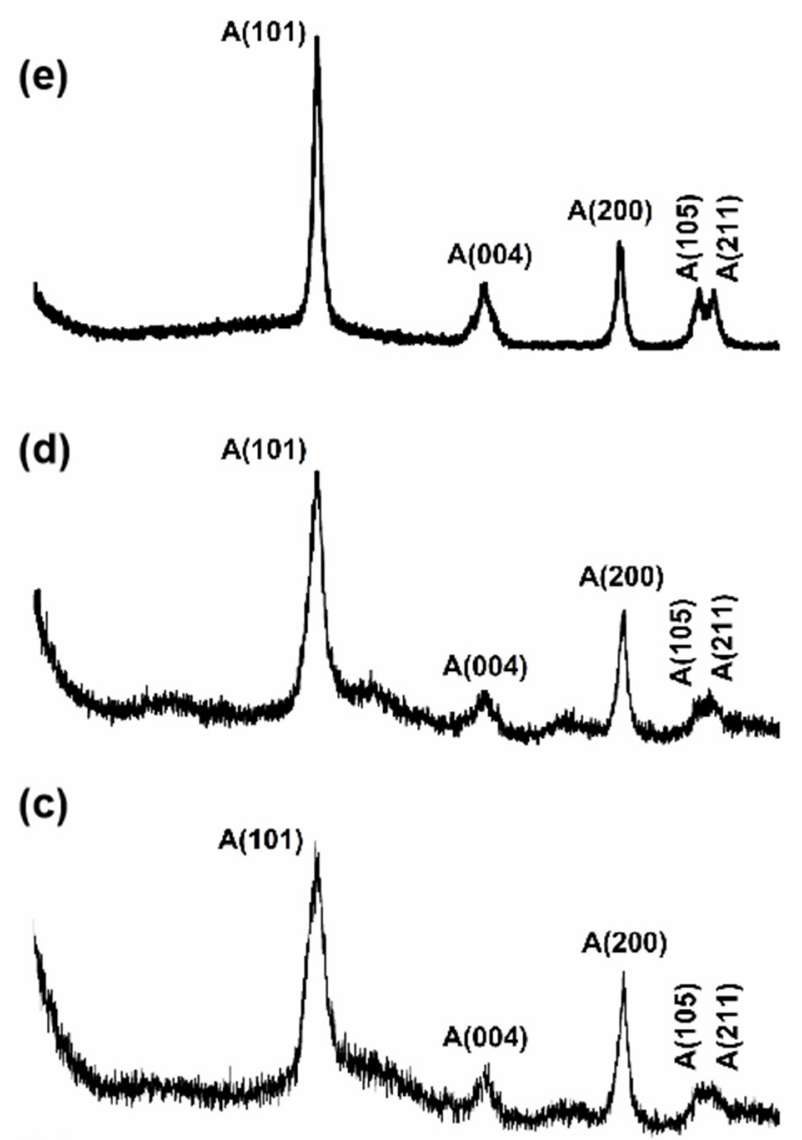

(b)

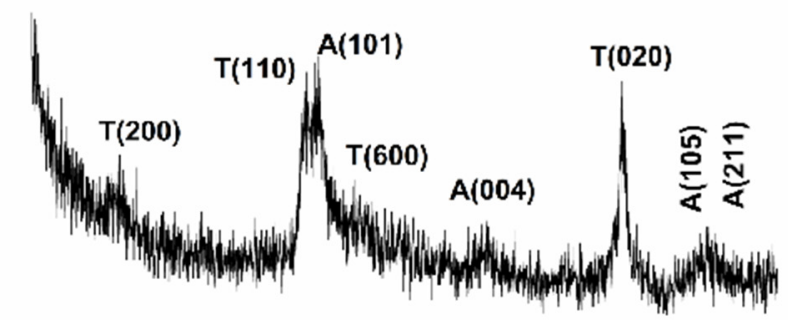

(a)

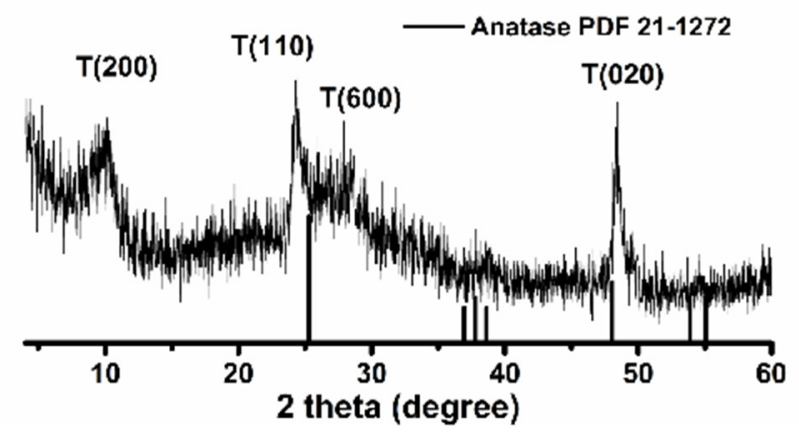

Figure 1. $\mathrm{XRD}$ patterns of (a) $\mathrm{TiO}_{2}-60$ (the as-prepared $\mathrm{H}$-titanate tubes), (b) $\mathrm{TiO}_{2}-100$, (c) $\mathrm{TiO}_{2}-200$, (d) $\mathrm{TiO}_{2}-300,(e) \mathrm{TiO}_{2}-500, \mathrm{~T}$ is the $\mathrm{H}$-titanate phase and $\mathrm{A}$ is the anatase phase. 


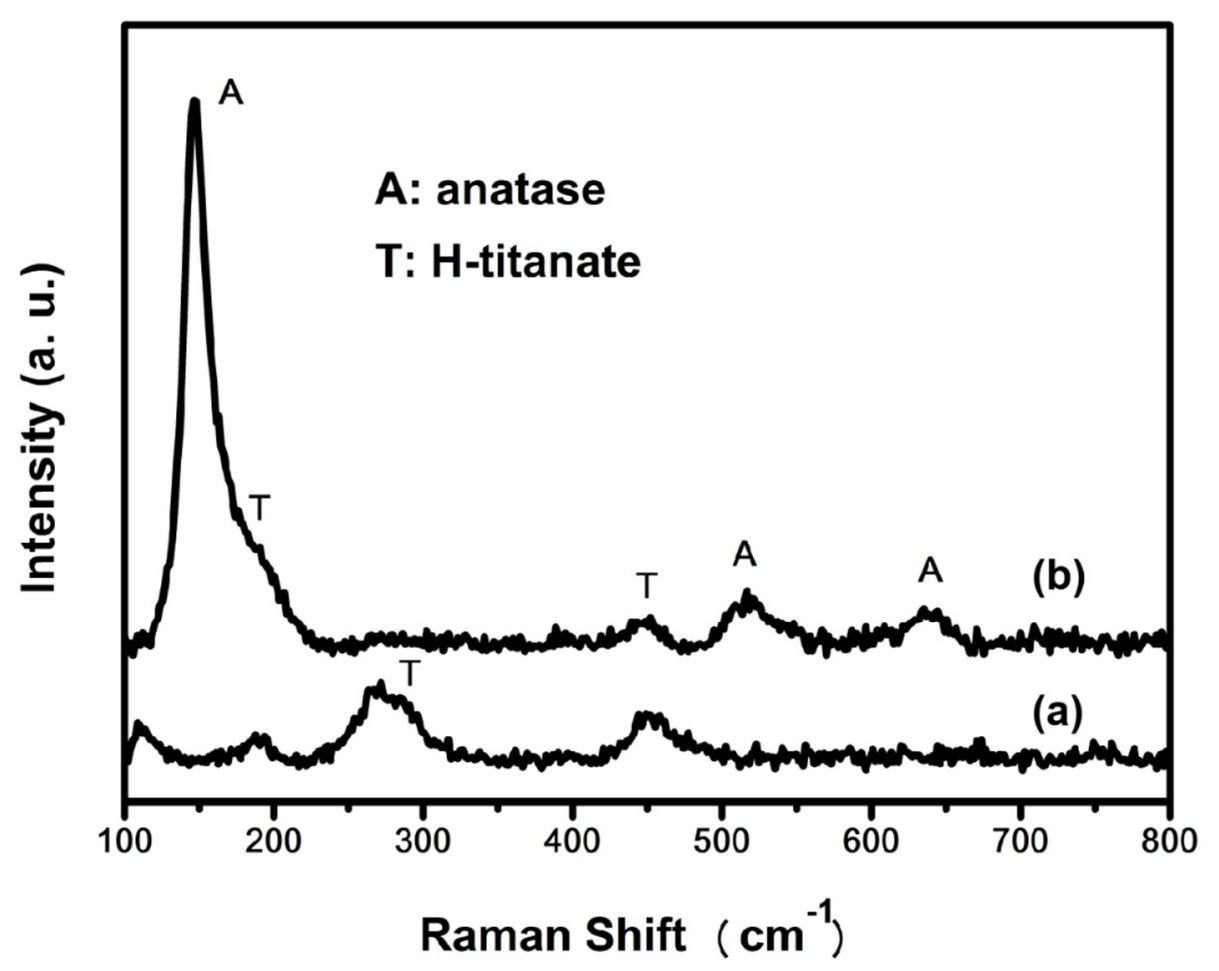

Figure 2. Raman spectra of (a) $\mathrm{TiO}_{2}-60$ and (b) $\mathrm{TiO}_{2}-100$.

The detailed characterization and crystal structure of the H-titanate/anatase composite were investigated via transmission electron microscopy (TEM), as shown in Figure 3. H-titanate was formed via the hydrothermal reaction of titanium powder with a concentrated $\mathrm{NaOH}$ solution to form sodium titanate and then a subsequent ion exchange reaction with $\mathrm{HNO}_{3}$ solution at room temperature. The dual-phase $\mathrm{H}$-titanate/anatase catalysts were prepared by in situ generation of anatase nanoparticles on the $\mathrm{H}$-titanate tube surfaces by a controllable surface dehydration reaction at a low drying temperature $\left(100^{\circ} \mathrm{C}\right)$. Figure S1a shows images of only the nanotubes, whereas Figure $3 \mathrm{~b}$ shows that some nanoparticles adhered to the surface of nanotubes, which indicates that a new phase was obtained after drying at $100{ }^{\circ} \mathrm{C}$. The average diameter of the as-synthesized nanoparticles is about $5-10 \mathrm{~nm}$. Figure $3 \mathrm{~b} 1$ (high-resolution transmission electron microscopy (HRTEM) images) distinctly reveals a lattice fringe spacing of $0.35 \mathrm{~nm}$, which corresponds well with the (101) anatase. Figure $3 \mathrm{~b} 2$ shows that the nanotubes are crystallized of layered $\mathrm{H}$-titanate. It also indicates that the $\mathrm{H}$-titanate retains its morphology of nanotubes, and the anatase reveals the morphology of nanoparticles. There are only particles in Figure S1b, which indicates that when the calcination temperature increased to $500{ }^{\circ} \mathrm{C}, \mathrm{H}$-titanate nanotubes completely transformed into anatase nanoparticles. The above results coincide with the XRD analysis. At a low drying temperature $\left(100^{\circ} \mathrm{C}\right)$, the photocatalyst retained its original nanotube structure and a small amount of anatase nanoparticles were formed on the H-titanate nanotube surfaces. Specific surface area (BET) is a key factor in photodegradation. High specific surface area offers more reaction sites for dye molecules and hydroxyl groups. Therefore, the porosities of the samples were determined by $\mathrm{N}_{2}$ sorption. Figure $3 c$ exhibits the $\mathrm{N}_{2}$ sorption isotherms of all as-synthesized products and the DFT (Density Functional Theory) pore size distributions of the dual-phase photocatalyst. The obtained isotherms of the samples are the typical IUPAC type-IV isotherm. We can see that the isotherms decreased with the decrease in the amount of H-titanate nanotubes. The corresponding DFT pore size distribution curve (inset of Figure 3c) of the dual-phase catalyst shows two pore sizes centered at $\sim 1.0 \mathrm{~nm}$ and $\sim 12 \mathrm{~nm}$, respectively, which directly proves the layered and nanotubed structure of the dual phase catalyst. BET measurements show that the as-synthesized $\mathrm{H}$-titanate tubes have a surface area of $245 \mathrm{~m}^{2} \cdot \mathrm{g}^{-1}$, and dual-phase photocatalyst has a surface area of $174 \mathrm{~m}^{2} \cdot \mathrm{g}^{-1}$. As the calcination temperature raised to $500^{\circ} \mathrm{C}$, the surface area of the 
product decreased to $95 \mathrm{~m}^{2} \cdot \mathrm{g}^{-1}$ (Table S1). The decrease in surface area may be due to the decreased proportion of layered H-titanate nanotubes. In other words, the specific surface area of the catalysts is enormously increased by the H-titanate nanotubes, which is hoping to enhance the photocatalytic property of the nanomaterials.
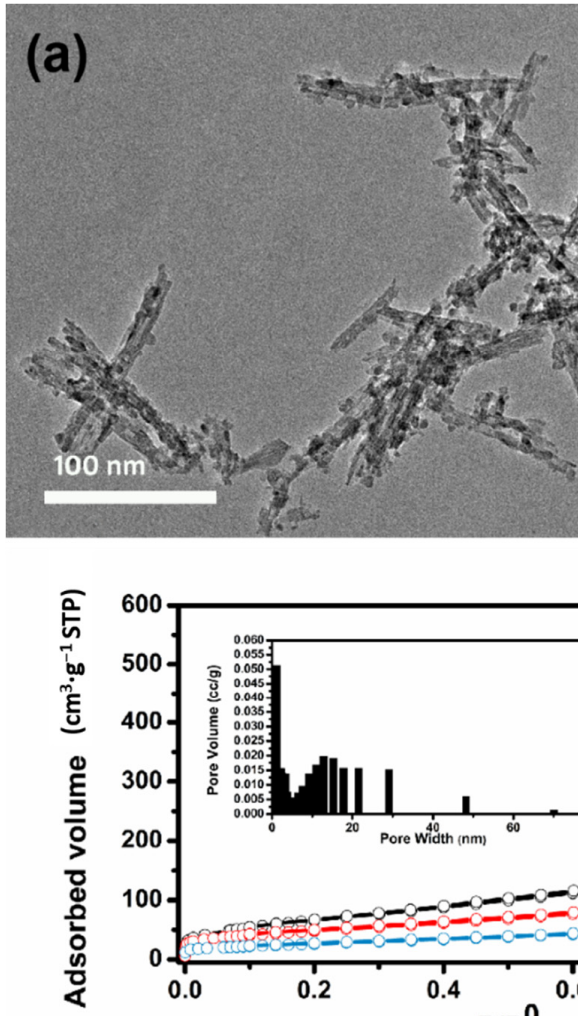

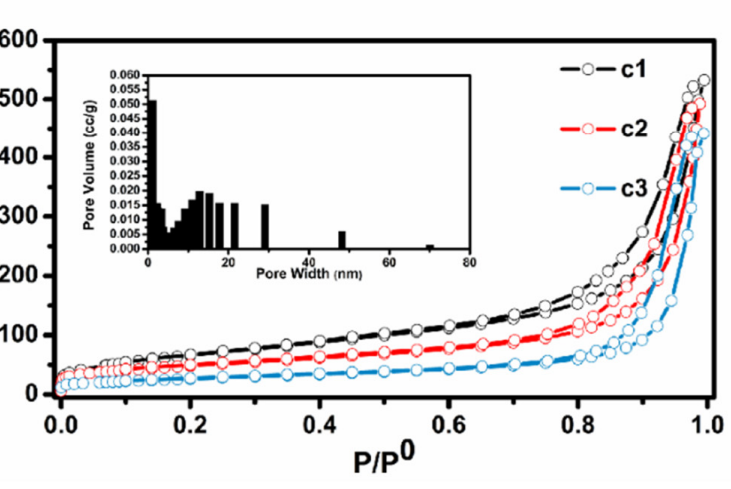

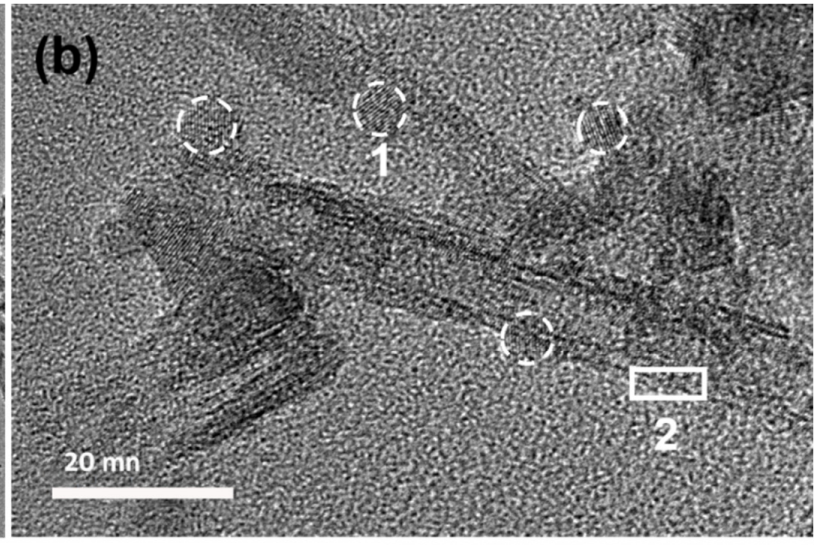

(c)

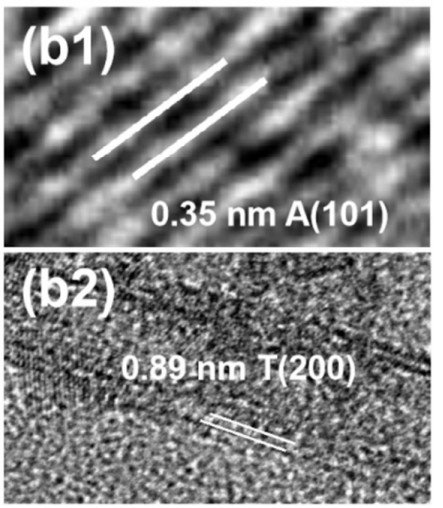

Figure 3. (a,b) are the TEM images of $\mathrm{TiO}_{2}-100$. (b1) and (b2) are the HRTEM images of $\mathrm{TiO}_{2}-100$ for the enlarged view of the circle and rectangle areas in (b). (c) Reversible nitrogen gas adsorption isotherm for the (c1) $\mathrm{TiO}_{2}-60$, (c2) $\mathrm{TiO}_{2}-100$, (c3) $\mathrm{TiO}_{2}-500$ measured at $77 \mathrm{~K}$. The inset is the pore size distribution of the $\mathrm{TiO}_{2}-100$ photocatalyst calculated via DFT method.

UV-Vis diffuse reflectance absorption spectra of as-synthesized H-titanate nanotubes $\left(\mathrm{TiO}_{2}-60\right)$, dual-phase H-titanate/anatase $\left(\mathrm{TiO}_{2}-100\right)$ and anatase nanoparticles $\left(\mathrm{TiO}_{2}-500\right)$ are shown in the Figure 4a. As indicated in Figure 4a, in the range of 200-800 nm, the H-titanate nanotubes, dual-phase H-titanate/anatase and anatase samples demonstrate similar absorption. Compared with that of pure H-titanate and anatase, the absorption edge of dual-phase H-titanate/anatase moved toward a longer wavelength. It may be owing to the synergistic effect of $\mathrm{H}$-titanate and anatase, resulting in a narrow band gap, which is a crucial role to the realization of solar energy conversion. The Kubelka-Munk method is often used to estimate the band gap energy $\left(\mathrm{E}_{\mathrm{g}}\right)$ of as-prepared products [23]. Figure $4 \mathrm{~b}$ reveals the Tauc plots of $(\alpha \mathrm{h} v)^{2}$ vs. photon energy (hv) of H-titanate tubes, dual-phase H-titanate/anatase and anatase photocatalyst. The band gap $\left(E_{g}\right)$ can be acquired by extending the vertical segment to the $\mathrm{h} v$ axis. As shown in Figure $4 \mathrm{~b}$, the $\mathrm{E}_{\mathrm{g}}$ of the H-titanate, H-titanate/anatase, and anatase is 3.48, 3.30, and $3.36 \mathrm{eV}$, respectively, which reveals the same results with the ultraviolet-visible diffffuse reflectance spectra (UV-Vis DRS) analysis. The photoluminescence (PL) technique is usually used to investigate the charge carrier separation and transfer processes because PL emission results from the free photogenerated carriers recombination [24]. Figure 4c shows the PL spectra of dual-phase H-titanate/anatase, H-titanate and anatase excited at $315 \mathrm{~nm}$. The emission intensity of dual-phase $\mathrm{H}$-titanate/anatase is much weaker than that of pure H-titanate and anatase, which can be attributed to the formation of H-titanate/anatase heterojunction in two semiconductor interfaces. Different band edge 
positions of $\mathrm{H}$-titanate and anatase can reduce the recombination of the carriers [19,25]. That enables more free electrons and holes to participate in the photocatalytic reactions and accelerates the photocatalytic process.
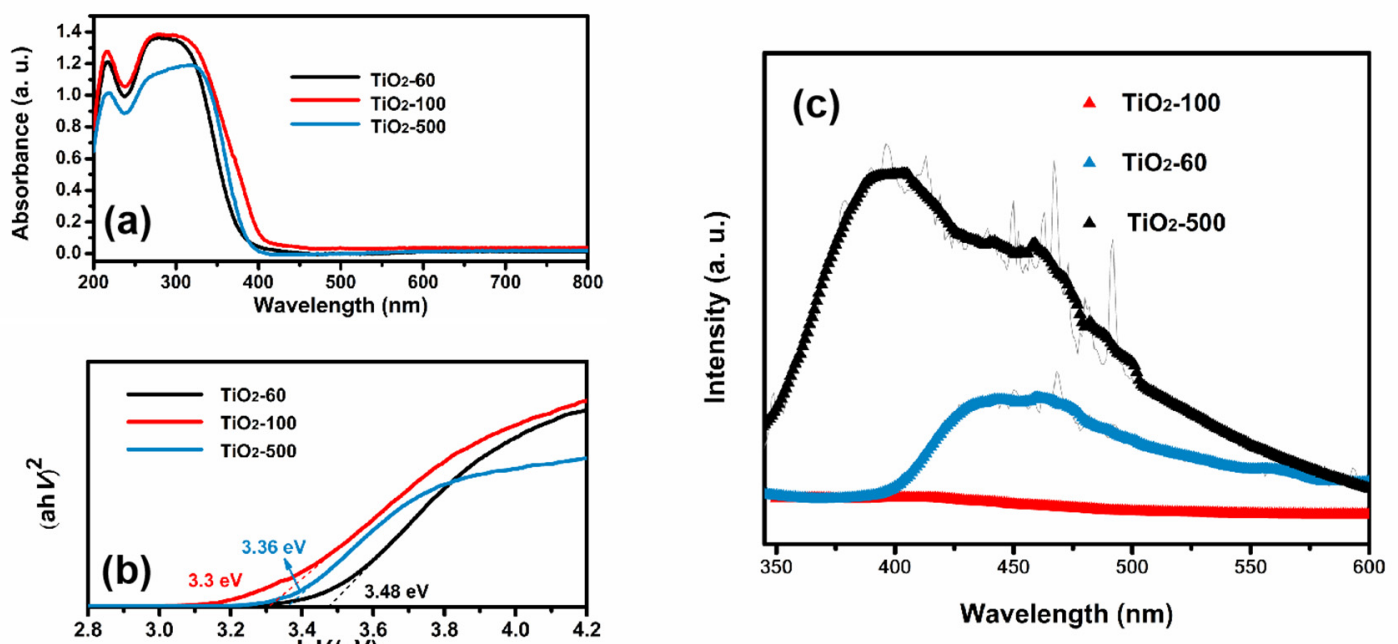

Figure 4. (a) UV/Vis diffuse reflectance absorption spectra, (b) plots of $(\alpha \mathrm{h} v)^{2}$ versus $\mathrm{h} v$, and (c) PL spectra of $\mathrm{TiO}_{2}-60, \mathrm{TiO}_{2}-100$ and $\mathrm{TiO}_{2}-500$.

The photocatalytic activities of as-prepared samples were studied by degradation of methyl orange $(\mathrm{MO})$, rhodamine $\mathrm{B}(\mathrm{RhB})$ and methylene blue $(\mathrm{MB})$ in aqueous solution under visible light at room temperature. In order to evaluate the photocatalytic efficiency, P25 was chosen as the photocatalytic reference material. In general, high specific surface area can improve the adsorption performance of the materials. Therefore, prior to the photocatalytic degradation studies, the adsorption properties of the materials were investigated. In general, a high degree of surface adsorption was observed within the first $30 \mathrm{~min}$ of stirring time in dark before attaining the saturation level. In Figure 5a and Figure S2A, P25 shows no obvious adsorption capacity, whereas the as-received dual-phase $\mathrm{H}$-titanate/anatase catalyst shows $90 \% \mathrm{MB}, 25 \% \mathrm{RhB}, 8.9 \% \mathrm{MO}$ dye adsorption, the $\mathrm{H}$-titanate shows $92 \% \mathrm{MB}, 59 \% \mathrm{RhB}, 9.1 \% \mathrm{MO}$ adsorption due to their nanotubed and layered structures, larger specific surface areas, which are beneficial for enhancing the photocatalytic activity. In addition, the electrostatic attraction between the catalysts and the dye molecules plays an important role in the different adsorption behavior of several dyes [26]. $\mathrm{MB}, \mathrm{RhB}$ is cationic and $\mathrm{MO}$ is anionic in the aqueous solution, while the nanotubes possess negative surface charge, which is more favorable to absorb $\mathrm{MB}$ and RhB. Although both RhB and MB are cationic dyes, the adsorption capacity of nanotubes for $\mathrm{MB}$ is much higher than that of RhB, which is due to the different molecular structures of dyes. The more linear shape and smaller size of MB molecule mean a weaker steric hindrance during the adsorption process [26]. In order to verify the adsorption of dye on H-titanate/anatase, FTIR analysis was carried out after a MB adsorption and degradation test (Figure S3). According to the previous report [27], in adsorption test, the peaks at $2926 \mathrm{~cm}^{-1}$ and $666 \mathrm{~cm}^{-1}$ indicated that the MB was adsorbed onto the $\mathrm{H}$-titanate/anatase nanotubes surfaces. After the degradation process, the peaks disappeared, suggesting that MB degraded completely. 

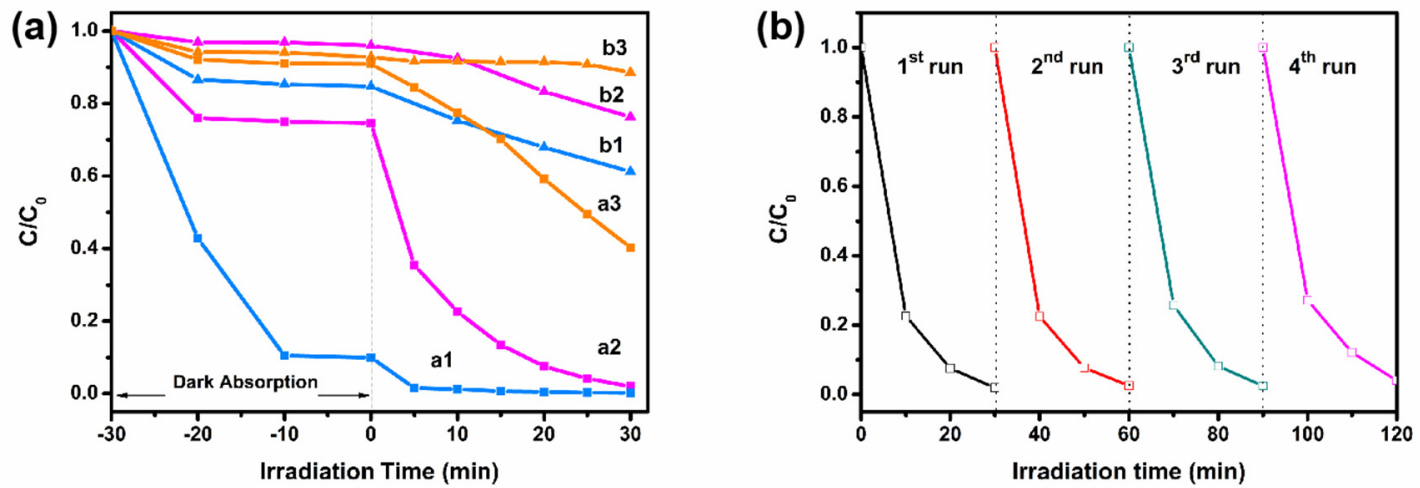

Figure 5. (a) Photocatalytic degradation of methylene blue (MB) (a1), rhodamine B (RhB) (a2), methyl orange (MO) (a3) over the dual-phase H-titanate/anatase catalysts and photocatalytic degradation of MB (b1), RhB (b2), MO (b3) over P25 under visible light irradiation, (b) cycling experiments of $\mathrm{H}$-titanate/anatase catalysts for $\mathrm{RhB}$ degradation under visible light irradiation.

When visible light was turned on, as shown in Figure $5 \mathrm{a}, \mathrm{RhB}$ was almost decomposed within only 30 min (Figure $5 \mathrm{a} 2$ and Figure S4), MO was removed by about $60 \%$ (Figure 5a3) within $30 \mathrm{~min}$ illumination, and the MB completely decomposed in just $5 \mathrm{~min}$ (Figure 5a1) in the test of the dual-phase H-titanate/anatase photocatalyst, whereas P25 showed no significant degradation for the dyes in $30 \mathrm{~min}$. Additionally, without any photocatalyst, $\mathrm{RhB}, \mathrm{MB}$ and $\mathrm{MO}$ were hardly degraded by visible light [28-30], which demonstrated the high reactivity of as-synthesized dual-phase nanotubes catalysts. Compared with many reported $\mathrm{TiO}_{2}$-based materials, it also shows higher photocatalytic activity for the degradation of organic dyes under visible light. Xiong et al. reported that under the visible light irradiation, $\mathrm{MB}$ degraded completely in about $175 \mathrm{~min}$ in the presence of the nitrogen-doped titanate-anatase core-shell nanobelts catalyst [14]. S-doped $\mathrm{Na}_{2} \mathrm{Ti}_{6} \mathrm{O}_{13} @ \mathrm{TiO}_{2}$ core-shell nanorods can completely decompose $\mathrm{MB}$ in 100 min under visible light [31]. Li et al. demonstrated that within 60 min of UV light irradiation, RhB was completely decomposed in the presence of the double-shell anatase-rutile $\mathrm{TiO}_{2}$ spheres [32]. The report of Pan et al. showed that within 40 min visible light irradiation, $\mathrm{MO}$ can be degraded by about $50 \%$ in the presence of $\mathrm{GQD}^{-\mathrm{TiO}_{2}}$ heterojunctions [33]. Figure S2A shows the photocatalytic activity of all as-synthesized catalysts for $\mathrm{MB}, \mathrm{RhB}$ and $\mathrm{MO}$ under visible light irradiation. Compared with pure $\mathrm{H}$-titanate and anatase catalysts, the dual-phase catalyst exhibited the best photoactivity. Figure S2B displays the degradation rate of the materials (dual-phase $\mathrm{H}$-titanate/anatase and P25) for MO, RhB under visible light irradiation. It is noted that the dual-phase catalyst displays better degradation efficiency than P25. H-titanate/anatase photocatalyst shows 15 times higher efficiency for the photodegradation of RhB and 17 times higher efficiency for the photodegradation of MO compared to P25 under visible light radiation, which demonstrated the high photocatalytic activity of the dual-phase H-titanate/anatase. In order to evaluate the stability of the dual-phase photocatalyst, several photodegradation tests of $\mathrm{RhB}$ under visible light were carried out (Figure 5b). After four cycles of photocatalytic degradation, within about $30 \mathrm{~min}$, the RhB could be decomposed completely, which indicates that the dual-phase H-titanate/anatase has excellent photocatalytic stability.

Based on above results, the dual-phase (H-titanate/anatase) catalyst reveals a high visible light photo-degradation ability. It can be expounded by the following involved reasons: (1) In-Situ generation can form a strong interfacial coupling between $\mathrm{H}$-titanate and anatase, which is useful for accelerating charge transfer and improving the photocatalytic activity [17]; (2) The dual-phase catalyst retains the nanotubed and layered structures, and possesses a high BET surface area. Large specific surface area and pore structure can provide more active sites to adsorb organic pollutants and promote the diffusion of organic molecules inside the pores. The layered titanate product has been considered as an excellent adsorbent $[34,35]$. It would be beneficial to improve the photocatalytic activity. In addition, the meso-nanotubes structure is also conducive to the rapid diffusion of quantum, which is formed in the 
photocatalytic process, further promoting photogenerated charge transport to improve the separation rate [36,37]; (3) The synergetic effect between $\mathrm{H}$-titanate and anatase is one of the major ingredients for its enhanced visible light photocatalytic activity. When two phases combined, a staggered band gap was formed, which lead to the efficient charge separation of the cross phase junction [38]. As shown in Figure 6, under visible light irradiation, anatase in dual-phase can be excited to generate electron-hole pairs. Electrons are excited from the valence band (VB) to the conduction band (CB). According to the energy band data, the $\mathrm{CB}$ and the VB energy potentials in anatase are -0.26 and $2.94 \mathrm{eV}$ [39], while those of titanate are -0.50 and $3.03 \mathrm{eV}$ [31]. The photogenerated electrons accumulated on anatase will migrate from the $\mathrm{CB}$ of anatase to that of titanate due to the potential difference. In this way, titanate can effectively collect photogenerated electrons and anatase collect holes. These electrons react with the surface adsorbed $\mathrm{O}_{2}$ to form $\mathrm{O}_{2}^{-} \cdot$, because the $\mathrm{CB}$ edge potential of titanate $(-0.50 \mathrm{eV})$ is more negative than the standard redox potential of $\mathrm{O}_{2} / \mathrm{O}_{2}^{-} \cdot(-0.33 \mathrm{eV})$ [40]. In the mean time, the VB potential of anatase $(2.94 \mathrm{eV})$ is more positive than the standard redox potential of $\cdot \mathrm{OH} / \mathrm{OH}^{-}(1.99 \mathrm{eV})[41,42]$, the holes react with $\mathrm{OH}^{-}$to generate $\cdot \mathrm{OH}$ radicals. Then the organic pollutants could be mineralized by the produced $\cdot \mathrm{OH}$ and $\mathrm{O}_{2}{ }^{-}$. radicals. Hence, the above efficient separation of photogenerated electron-hole pairs process improves the photodegradation rate of dyes; (4) The new anatase phase with a small average crystal size means a stronger redox ability because of the quantum size effect [43]. Combining all above factors, the dual-phase $\mathrm{H}$-titanate/anatase photocatalysts displayed high visible light activity.

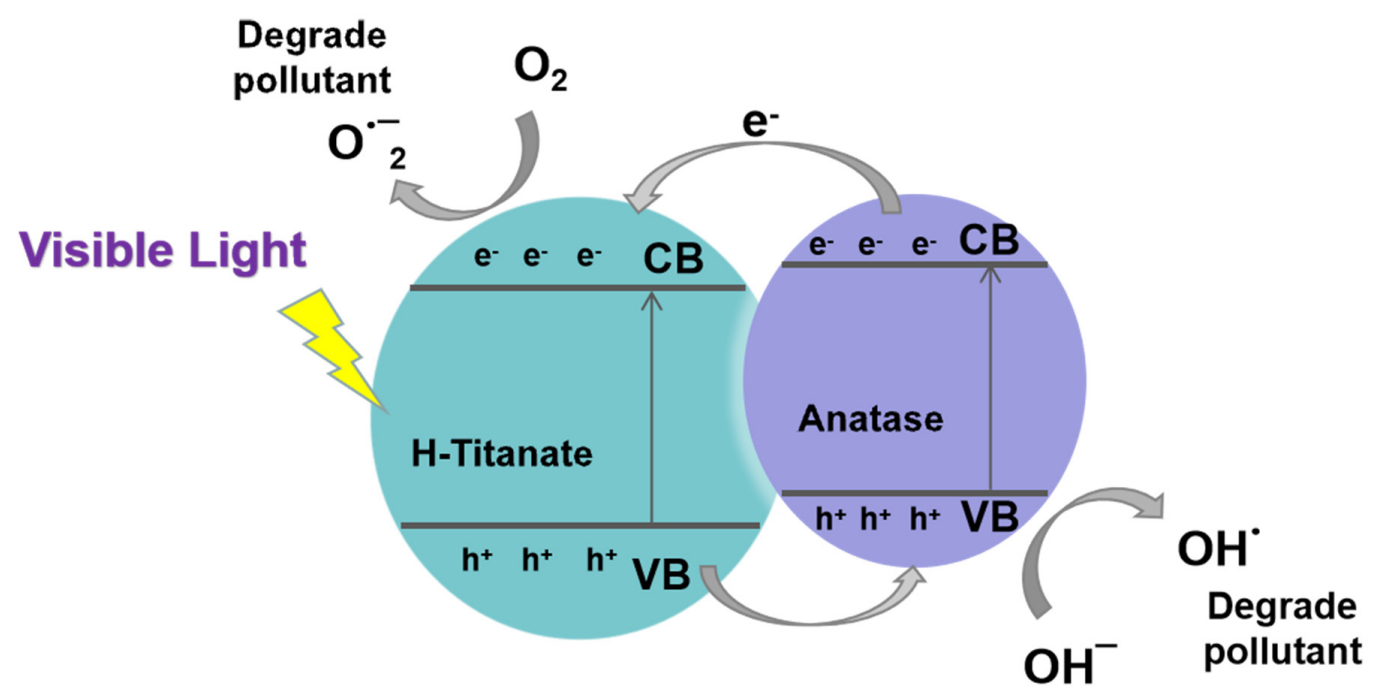

Figure 6. The proposed photocatalytic mechanism of H-titanate/anatase composite.

\section{Materials and Methods}

\subsection{Materials}

The titanium powder was purchased from Aladdin, Tianjin, China. The $\mathrm{NaOH}$ and the $\mathrm{HNO}_{3}$ were purchased from Sinopharm Chemical Reagent Co., Ltd., Shanghai, China.

\subsection{Catalyst Preparation}

The photocatalysts were prepared by the following synthetic route. Firstly, $1.5 \mathrm{~g}$ titanium powder was mixed with $35 \mathrm{~mL} \mathrm{NaOH}$ solution $(10 \mathrm{M})$ and stirred at room temperature for $5 \mathrm{~h}$. The mixture was transferred into a Teflon-lined autoclave and kept at $150{ }^{\circ} \mathrm{C}$ for $72 \mathrm{~h}$. The obtained precipitates were washed with deionized water until neutral and dried in an oven $\left(60^{\circ} \mathrm{C}\right)$ overnight. Afterwards, the ion-exchange reaction was followed with $0.5 \mathrm{M} \mathrm{HNO}_{3}$ solution for $3 \mathrm{~h}$ at room temperature. There were three times ion-exchange reactions for the product. The final product was dried at $60^{\circ} \mathrm{C}$ 
for $24 \mathrm{~h}$ to produce the hydrogen titanate (H-titanate) tubes and then dried at $100{ }^{\circ} \mathrm{C}$ for $24 \mathrm{~h}$ to yield hydrogen titanate/anatase nanotubes. The as-prepared H-titanate was calcined in air at 200, 300 and $500{ }^{\circ} \mathrm{C}$ for $5 \mathrm{~h}$ to get $\mathrm{TiO}_{2}$ products. Henceforth, these samples are referred to as $\mathrm{TiO}_{2}-60, \mathrm{TiO}_{2}-100$, $\mathrm{TiO}_{2}-200, \mathrm{TiO}_{2}-300$ and $\mathrm{TiO}_{2}-500$, respectively.

\subsection{Catalyst Characterization}

X-ray powder diffraction (XRD) analysis was carried out using a D/Max-2550 X-ray powder diffractometer (Tokyo, Japan) with $\mathrm{Cu} \mathrm{K} \alpha$ radiation. The ultraviolet-visible diffuse reflectance spectra of the samples were measured on a UV-Vis-NIR spectrophotometer (Shimadzu U-4100, Shanghai, China) detecting absorption over the range of 200-800 $\mathrm{nm}$. The morphologies of samples were measured on a Tecnai G2 S-Twin F20 transmission electron microscopy (TEM, FEI, Hillsboro, FL, USA). $\mathrm{N}_{2}$ adsorption-desorption isotherms were obtained at $77 \mathrm{~K}$ on a Micromeritics ASAP 2020 sorptometer (Norcross, GA, USA). Raman spectra were recorded using a Renishaw InVia Raman spectrometer (London, UK) with a wavelength of $532 \mathrm{~nm}$. Room temperature photoluminescence (PL) spectra with an excitation wavelength of $315 \mathrm{~nm}$ were measured on a FLUOROMAX-4 (Beijing, China).

\subsection{Photocatalytic Activity Test}

The photocatalytic activities of the photocatalysts were performed at room temperature in a glass reactor fitted with a Xe lamp ( $300 \mathrm{~W})$. A $420 \mathrm{~nm}$ cut-on filter was used to ensure that only visible light illuminated the photocatalyst. The reaction liquid was prepared by mixing $0.25 \mathrm{~g}$ photocatalysts and $100 \mathrm{~mL}$ rhodamine $B$ (or $10 \mathrm{mg} / \mathrm{L} \mathrm{MB} ; 10 \mathrm{mg} / \mathrm{L} \mathrm{MO}$ ) aqueous solution $(10 \mathrm{mg} / \mathrm{L}$ ). Then, the suspension was stirred in dark for $30 \mathrm{~min}$ to reach adsorption-desorption equilibrium before irradiation. Then it was irradiated under visible light. The suspension $(8 \mathrm{~mL})$ was withdrawn from the irradiated solution at preset time intervals and centrifuged to separate the photocatalyst particles, and then the supernatants were analyzed by UV-Vis spectrophotometer (UV-2450, Shanghai, China).

\section{Conclusions}

In conclusion, anatase nanoparticles generated on $\mathrm{H}$-titanate nanotubes surfaces were successfully synthesized by an ingenious method. H-titanate tubes were prepared by a hydrothermal synthesis of Ti powder in concentrated $\mathrm{NaOH}$ solution and an ion exchange process with $\mathrm{HNO}_{3}$ solution. After that, at a relatively low drying temperature, a small amount of anatase nanoparticles were in-situ formed on the surface of the $\mathrm{H}$-titanate tubes by the surface dehydration reaction. It showed higher photocatalytic activity than pure $\mathrm{H}$-titanate nanotube, anatase, and P25 under visible light $(100 \%$ rhodamine B (RhB) and methylene blue (MB), 60\% methyl orange (MO) degraded in 30 min under visible light irradiation) due to the in-situ transformation, the smaller average crystal size, the nanotubed and layered structure, the large BET surface areas and the synergistic effect of the $\mathrm{H}$-titanate/anatase dual phases, which can accelerate the transfer of electron-hole pairs and inhibit their recombination. This work provides an ingenious and simple method to prepare an efficient visible-light-responsive $\mathrm{TiO}_{2}$-based photocatalyst for solving environment problems.

Supplementary Materials: The following are available online at http://www.mdpi.com/2073-4344/10/6/695/s1, Figure S1: TEM images of (a) $\mathrm{TiO}_{2}-60$, (b) $\mathrm{TiO}_{2}-500$, Figure S2: (A) Photocatalytic degradation of RhB over the $\left(a_{1}\right)$ dual-phase H-titanate/anatase, $\left(a_{2}\right) \mathrm{H}$-titanate, $\left(a_{3}\right)$ anatase catalysts; photocatalytic degradation of $M B$ over the $\left(b_{1}\right)$ dual-phase H-titanate/anatase, $\left(b_{2}\right)$ H-titanate, $\left(b_{3}\right)$ anatase catalysts; photocatalytic degradation of $\mathrm{MO}$ over the $\left(\mathrm{c}_{1}\right)$ dual-phase H-titanate/anatase, $\left(\mathrm{c}_{2}\right)$ H-titanate, $\left(c_{3}\right)$ anatase catalysts under visible light irradiation. (B) Photocatalytic kinetic plot of the $\left(a_{1}\right)$ P25, $\left(a_{2}\right)$ dual-phase H-titanate/anatase for degradation of RhB; photocatalytic kinetic plot of the $\left(b_{1}\right)$ P25, $\left(b_{2}\right)$ dual-phase H-titanate/anatase for degradation of MO under visible light irradiation.

Author Contributions: Z.Z. and W.F. conceived and designed the experiments; Funding acquisition, L.Z.; Z.S., H.B., J.D., Z.L., F.L. and D.Z. performed the experiments and analyzed the data; W.F. wrote the paper. All authors have read and agreed to the published version of the manuscript. 
Funding: This work was supported by the Science and Technology Development Planning of Jilin Province, China (No. 20170101098JC).

Conflicts of Interest: The authors declare no conflict of interest.

\section{References}

1. Cao, S.W.; Low, J.X.; Yu, J.G.; Jaroniec, M. Polymeric Photocatalysts Based on Graphitic Carbon Nitride. Adv. Mater. 2015, 27, 2150-2176. [CrossRef]

2. Zhang, X.; Chen, Y.; Xiao, Y.; Zhou, W.; Tian, G.; Fu, H. Enhanced charge transfer and separation of hierarchical hydrogenated $\mathrm{TiO}_{2}$ nanothorns/carbon nanofibers composites decorated by NiS quantum dots for remarkable photocatalytic $\mathrm{H}_{2}$ production activity. Nanoscale 2018, 10, 4041-4050. [CrossRef]

3. Dai, G.; Qin, H.; Zhou, H.; Wang, W.; Luo, T. Template-free fabrication of hierarchical macro/mesoporpous $\mathrm{SnS}_{2} / \mathrm{TiO}_{2}$ composite with enhanced photocatalytic degradation of Methyl Orange (MO). Appl. Surf. Sci. 2018, 430, 488-495. [CrossRef]

4. Lyu, J.; Gao, J.; Zhang, M.; Fu, Q.; Sun, L.; Hu, S.; Zhong, J.; Wang, S.; Li, J. Construction of homojunction-adsorption layer on anatase $\mathrm{TiO}_{2}$ to improve photocatalytic mineralization of volatile organic compounds. Appl. Catal. B 2017, 202, 664-670. [CrossRef]

5. Meng, A.; Zhang, L.; Cheng, B.; Yu, J. TiO $2-\mathrm{MnOx}-\mathrm{Pt}$ Hybrid Multiheterojunction Film Photocatalyst with Enhanced Photocatalytic $\mathrm{CO}_{2}$-Reduction Activity. ACS Appl. Mater. Interfaces 2018, 11, 5581-5589. [CrossRef] [PubMed]

6. Shi, Y.; Li, H.; Wang, L.; Shen, W.; Chen, H. Novel $\alpha$-Fe2O3/CdS Cornlike Nanorods with Enhanced Photocatalytic Performance. ACS Appl. Mater. Interfaces 2012, 4, 4800-4806. [CrossRef] [PubMed]

7. Ran, J.; Jaroniec, M.; Qiao, S.Z. Cocatalysts in Semiconductor-based Photocatalytic $\mathrm{CO}_{2}$ Reduction: Achievements, Challenges, and Opportunities. Adv. Mater. 2018, 30, 1704649(1)-1704649(31). [CrossRef]

8. Fu, W.W.; Li, G.D.; Wang, Y.; Zeng, S.J.; Yan, Z.J.; Wang, J.W.; Xin, S.G.; Zhang, L.; Wu, S.W.; Zhang, Z.T. Facile formation of mesoporous structured mixed-phase (anatase/rutile) $\mathrm{TiO}_{2}$ with enhanced visible light photocatalytic activity. Chem. Commun. 2018, 54, 58-61. [CrossRef]

9. Meng, A.; Zhang, L.; Cheng, B.; Yu, J. Dual Cocatalysts in $\mathrm{TiO}_{2}$ Photocatalysis. Adv. Mater. 2019, 31, 1807660(1)-1807660(31).

10. Niu, S.; Zhang, R.; Zhang, Z.; Zheng, J.; Jiao, Y.; Guo, C. In situ construction of the BiOCl/ $\mathrm{Bi}_{2} \mathrm{Ti}_{2} \mathrm{O}_{7}$ heterojunction with enhanced visible-light photocatalytic activity. Inorg. Chem. Front. 2019, 6, 791-798. [CrossRef]

11. Bavykin, D.V.; Friedrich, J.M.; Walsh, F.C. Protonated Titanates and $\mathrm{TiO}_{2}$ Nanostructured Materials: Synthesis, Properties, and Applications. Adv. Mater. 2006, 18, 2807-2824. [CrossRef]

12. Mao, Y.; Wong, S.S. Size- and Shape-Dependent Transformation of Nanosized Titanate into Analogous Anatase Titania Nanostructures. J. Am. Chem. Soc. 2006, 128, 8217-8226. [CrossRef] [PubMed]

13. Buchholcz, B.; Haspel, H.; Kukovecz, Á.; Kónya, Z. Low-temperature conversion of titanate nanotubes into nitrogen-doped $\mathrm{TiO}_{2}$ nanoparticles. CrystEngComm 2014, 16, 7486-7492. [CrossRef]

14. Xiong, Z.; Zhao, X. Nitrogen-doped titanate-anatase core-shell nanobelts with exposed $\{101\}$ anatase facets and enhanced visible light photocatalytic activity. J. Am. Chem. Soc. 2012, 134, 5754-5757. [CrossRef]

15. Yan, Y.; Qiu, X.; Wang, H.; Li, L.; Fu, X.; Wu, L.; Li, G. Synthesis of titanate/anatase composites with highly photocatalytic decolorization of dye under visible light irradiation. J. Alloys Compd. 2008, 460, 491-495. [CrossRef]

16. Harsha, N.; Ranya, K.R.; Babitha, K.B.; Shukla, S.; Biju, S.; Reddy, M.L.P.; Warrier, K.G.K. Hydrothermal Processing of Hydrogen Titanate/Anatase-Titania Nanotubes and Their Application as Strong Dye-Adsorbents. J. Nanosci. Nanotechnol. 2010, 10,1-13. [CrossRef]

17. Wang, P.; Yi, X.; Lu, Y.; Yu, H.; Yu, J. In-situ synthesis of amorphous $\mathrm{H}_{2} \mathrm{TiO}_{3}$-modified $\mathrm{TiO}_{2}$ and its improved photocatalytic $\mathrm{H}_{2}$-evolution performance. J. Colloid Interface Sci. 2018, 532, 272-279. [CrossRef]

18. Cheng, Y.H.; Huang, Y.Z.; Kanhere, P.D.; Subramaniam, V.P.; Gong, D.G.; Zhang, S.; Highfield, J.; Schreyer, M.K.; Chen, Z. Dual-Phase Titanate/Anatase with Nitrogen Doping for Enhanced Degradation of Organic Dye under Visible Light. Chem. Eur. J. 2011, 17, 2575-2578. [CrossRef] 
19. Cai, J.; Zhu, Y.; Liu, D.; Meng, M.; Hu, Z.; Jiang, Z. Synergistic Effect of Titanate-Anatase Heterostructure and Hydrogenation-Induced Surface Disorder on Photocatalytic Water Splitting. ACS Catal. 2015, 5, 1708-1716. [CrossRef]

20. Zhang, L.; Lin, H.; Wang, N.; Lin, C.; Li, J. The evolution of morphology and crystal form of titanate nanotubes under calcination and its mechanism. J. Alloys Compd. 2007, 431, 230-235. [CrossRef]

21. Xiong, Z.; Dou, H.; Pan, J.; Ma, J.; Xu, C.; Zhao, X.S. Synthesis of mesoporous anatase $\mathrm{TiO}_{2}$ with a combined template method and photocatalysis. CrystEngComm 2010, 12, 3455-3457. [CrossRef]

22. Pant, B.; Park, M.; Park, S.-J. TiO2 NPs Assembled into a Carbon Nanofifiber Composite Electrode by a One-Step Electrospinning Process for Supercapacitor Applications. Polymers 2019, 11, 899. [CrossRef] [PubMed]

23. Nowak, M.; Kauch, B.; Szperlich, P. Determination of energy band gap of nanocrystalline SbSI using diffuse reflectance spectroscopy. Rev. Sci. Instrum. 2009, 80, 046107(1)-046107(3). [CrossRef] [PubMed]

24. Zhang, W.F.; Zhang, M.S.; Yin, Z.; Chen, Q. Photoluminescence in anatase titanium dioxide nanocrystals. Appl. Phys. B Laser Opt. 2000, 70, 261-265. [CrossRef]

25. Ng, J.; Xu, S.; Zhang, X.; Yang, H.Y.; Sun, D.D. Hybridized Nanowires and Cubes: A Novel Architecture of a Heterojunctioned $\mathrm{TiO}_{2} / \mathrm{SrTiO}_{3}$ Thin Film for Efficient Water Splitting. Adv. Funct. Mater. 2010, 20, 4287-4294. [CrossRef]

26. Wang, Q.; Zhang, B.; Lu, X.; Zhang, X.; Zhu, H.; Li, B. Multifunctional 3D $\mathrm{K}_{2} \mathrm{Ti}_{6} \mathrm{O}_{13}$ nanobelt-built architectures towards wastewater remediation: Selective adsorption, photodegradation, mechanism insight and photoelectrochemical investigation. Catal. Sci. Technol. 2018, 8, 6180-6195. [CrossRef]

27. Pant, B.; Ojha, G.P.; Kim, H.-Y.; Park, M.; Park, S.-J. Fly-ash-incorporated electrospun zinc oxide nanofifibers: Potential material for environmental remediation. Environ. Pollut. 2019, 245, 163-172. [CrossRef]

28. Fu, W.; Ding, S.; Wang, Y.; Wu, L.; Zhang, D.; Pan, Z.; Wang, R.; Zhang, Z.; Qiu, S. F, Ca co-doped TiO 2 nanocrystals with enhanced photocatalytic activity. Dalton Trans. 2014, 43, 16160-16163. [CrossRef]

29. Nguyen-Le, M.-T.; Lee, B.-K. Novel fabrication of a nitrogen-doped mesoporous $\mathrm{TiO}_{2}$-nanorod titanate heterojunction to enhance the photocatalytic degradation of dyes under visible light. RSC Adv. 2016, 6, 31347-31350.

30. Niu, F.; Chen, D.; Qin, L.; Zhang, N.; Wang, J.; Chen, Z.; Huang, Y. Facile Synthesis of Highly Efficient p-n Heterojunction $\mathrm{CuO} / \mathrm{BiFeO}_{3}$ Composite Photocatalysts with Enhanced Visible-Light Photocatalytic Activity. ChemCatChem 2015, 7, 3279-3289. [CrossRef]

31. Liu, C.; Liang, J.-Y.; Han, R.-R.; Wang, Y.-Z.; Zhao, J.; Huang, Q.-J.; Chen, J.; Hou, W.-H. S-doped $\mathrm{Na}_{2} \mathrm{Ti}_{6} \mathrm{O}_{13} @ \mathrm{TiO}_{2}$ core-shell nanorods with enhanced visible light photocatalytic performance. Phys. Chem. Chem. Phys. 2015, 17, 15165-15172. [CrossRef] [PubMed]

32. Li, S.; Chen, J.; Zheng, F.; Li, Y.; Huang, F. Synthesis of the double-shell anatase-rutile $\mathrm{TiO}_{2}$ hollow spheres with enhanced photocatalytic activity. Nanoscale 2013, 5, 12150-12155. [CrossRef] [PubMed]

33. Pan, D.; Jiao, J.; Li, Z.; Guo, Y.; Feng, C.; Liu, Y.; Wang, L.; Wu, M. Efficient Separation of Electron-Hole Pairs in Graphene Quantum Dots by $\mathrm{TiO}_{2}$ Heterojunctions for Dye Degradation. ACS Sustain. Chem. Eng. 2015, 3, 2405-2413. [CrossRef]

34. Tang, Y.; Lai, Y.; Gong, D.; Goh, K.-H.; Lim, T.-T.; Dong, Z.; Chen, Z. Ultrafast Synthesis of Layered Titanate Microspherulite Particles by Electrochemical Spark Discharge Spallation. Chem. Eur. J. 2010, 16, 7704-7708. [CrossRef]

35. Lim, Y.W.L.; Tang, Y.X.; Cheng, Y.H.; Chen, Z. Morphology, crystal structure and adsorption performance of hydrothermally synthesized titania and titanate nanostructures. Nanoscale 2010, 2, 2751-2757. [CrossRef] [PubMed]

36. Luo, Z.; Poyraz, A.S.; Kuo, C.-H.; Miao, R.; Meng, Y.; Chen, S.-Y.; Jiang, T.; Wenos, C.; Suib, S.L. Crystalline Mixed Phase (Anatase/Rutile) Mesoporous Titanium Dioxides for Visible Light Photocatalytic Activity. Chem. Mater. 2015, 27, 6-17. [CrossRef]

37. Gurulakshmi, M.; Selvaraj, M.; Selvamani, A.; Vijayan, P.; Sasi Rekha, N.R.; Shanthi, K. Enhanced visible-light photocatalytic activity of $\mathrm{V}_{2} \mathrm{O}_{5} / \mathrm{S}-\mathrm{TiO}_{2}$ nanocomposites. Appl. Catal. A 2012, 449, 31-46. [CrossRef]

38. Kho, Y.K.; Iwase, A.; Teoh, W.Y.; Ma“dler, L.; Kudo, A.; Amal, R. Photocatalytic $\mathrm{H}_{2}$ Evolution over $\mathrm{TiO}_{2}$ Nanoparticles. The Synergistic Effect of Anatase and Rutile. J. Phys. Chem. C 2010, 114, 2821-2829. [CrossRef] 
39. Meng, A.; Zhang, J.; Xu, D.; Cheng, B.; Yu, J. Enhanced photocatalytic $\mathrm{H}_{2}$-production activity of anatase $\mathrm{TiO}_{2}$ nanosheet by selectively depositing dual cocatalysts on $\{101\}$ and $\{001\}$ facets. Appl. Catal. B 2016, 198, 286-294. [CrossRef]

40. Zhang, D.; Wang, Q.; Wang, L.; Zhang, L. Magnetically separable $\mathrm{CdFe}_{2} \mathrm{O}_{4}$ /graphene catalyst and its enhanced photocatalytic properties. J. Mater. Chem. A 2015, 3, 3576-3585. [CrossRef]

41. Li, K.; Gao, S.; Wang, Q.; Xu, H.; Wang, Z.; Huang, B.; Dai, Y.; Lu, J. In-Situ-Reduced Synthesis of Ti ${ }^{3+}$ Self-Doped $\mathrm{TiO}_{2} / \mathrm{g}-\mathrm{C}_{3} \mathrm{~N}_{4}$ Heterojunctions with High Photocatalytic Performance under LED Light Irradiation. ACS Appl. Mater. Interfaces 2015, 7, 9023-9030. [CrossRef] [PubMed]

42. Yu, J.; Wang, S.; Low, J.; Xiao, W. Enhanced photocatalytic performance of direct Z-scheme g- $\mathrm{C}_{3} \mathrm{~N}_{4}-\mathrm{TiO}_{2}$ photocatalysts for the decomposition of formaldehyde in air. Phys. Chem. Chem. Phys. 2013, 15, 16883-16890. [CrossRef] [PubMed]

43. Liu, Z.Y.; Sun, D.D.; Guo, P.; Leckie, J.O. One-Step Fabrication and High Photocatalytic Activity of Porous $\mathrm{TiO}_{2}$ Hollow Aggregates by Using a Low-Temperature Hydrothermal Method Without Templates. Chem. Eur. J. 2007, 13, 1851-1855. [CrossRef] [PubMed]

(C) 2020 by the authors. Licensee MDPI, Basel, Switzerland. This article is an open access article distributed under the terms and conditions of the Creative Commons Attribution (CC BY) license (http://creativecommons.org/licenses/by/4.0/). 